\title{
Effects of solar wind magnetosphere coupling recorded at different geomagnetic latitudes: Separation of directly-driven and storage/release systems
}

Article

Published Version

Finch, I. D., Lockwood, M. L. and Rouillard, A. P. (2008) Effects of solar wind magnetosphere coupling recorded at different geomagnetic latitudes: Separation of directly-driven and storage/release systems. Geophysical Research Letters, 35 (21). L21105. ISSN 0094-8276 doi:

https://doi.org/10.1029/2008GL035399 Available at https://centaur.reading.ac.uk/7510/

It is advisable to refer to the publisher's version if you intend to cite from the work. See Guidance on citing.

Published version at: http://dx.doi.org/10.1029/2008GL035399

To link to this article DOI: http://dx.doi.org/10.1029/2008GL035399

Publisher: American Geophysical Union

All outputs in CentAUR are protected by Intellectual Property Rights law, including copyright law. Copyright and IPR is retained by the creators or other copyright holders. Terms and conditions for use of this material are defined in the End User Agreement. 


\section{www.reading.ac.uk/centaur}

\section{CentAUR}

Central Archive at the University of Reading

Reading's research outputs online 


\title{
Effects of solar wind magnetosphere coupling recorded at different geomagnetic latitudes: Separation of directly-driven and storage/ release systems
}

\author{
I. D. Finch, ${ }^{1}$ M. L. Lockwood, ${ }^{1,2}$ and A. P. Rouillard ${ }^{2}$ \\ Received 19 July 2008; revised 4 September 2008; accepted 12 September 2008; published 12 November 2008.
}

[1] The effect on geomagnetic activity of solar wind speed, compared with that of the strength of the interplanetary magnetic field, differs with geomagnetic latitude. In this study we construct a new index based on monthly standard deviations in the H-component of the geomagnetic field for all geomagnetic latitudes. We demonstrate that for this index the response at auroral regions correlates best with interplanetary coupling functions which include the solar wind speed while mid- and low-latitude regions respond to variations in the interplanetary magnetic field strength. These results are used to isolate the responsible geomagnetic current systems. Citation: Finch, I. D., M. L. Lockwood, and A. P. Rouillard (2008), Effects of solar wind magnetosphere coupling recorded at different geomagnetic latitudes: Separation of directly-driven and storage/release systems, Geophys. Res. Lett., 35, L21105, doi:10.1029/ 2008GL035399.

\section{Introduction}

[2] A large number of different geomagnetic indices are currently in use. The Dst, $A L$ and $A U$ indices are designed to monitor a specific current (respectively, the ring current, the northern hemisphere westward auroral electrojet and the northern hemisphere eastward auroral electrojet) [Mayaud, 1980, and references therein]. In contrast the planetary indices $-a a, a m, I H V, I D V$ and $m$ - are concerned with measuring general geomagnetic activity rather than any single magnetospheric phenomenon [Mayaud, 1980; Svalgaard et al., 2004; Svalgaard and Cliver, 2007; Rouillard et al., 2007; M. Lockwood et al., The long-term drift in geomagnetic activity: Calibration of the aa index using data from a variety of magnetometer stations, submitted to Annales Geophysicae, 2007.].

[3] The longest magnetometer-derived data sequence is that of the $a a$ index which is available continuously at 3hourly resolution from 1868. However, the $a a$ index (and the similarly derived am index) suffers from some subjectivity in its construction [see Mayaud, 1980, chapter 4.7]. It is thus impractical to reconstruct either of the $a a$ or $a m$ indices using readily available digital data because electronic derivation is impossible. It was in response to this problem, as brought to prominence by the recent debate about long-term trends in the a a index [Clilverd et al., 2002,

\footnotetext{
${ }^{1}$ Space Science and Technology Department, Rutherford Appleton Laboratory, Oxfordshire, UK

${ }^{2}$ Solar-Terrestrial Physics Group, Department of Physics and Astronomy, University of Southampton, Southampton, UK.
}

Copyright 2008 by the American Geophysical Union. 0094-8276/08/2008GL035399\$05.00
2005; Svalgaard et al., 2004; Svalgaard and Cliver, 2005, 2007; Jarvis, 2005; Martini and Mursula, 2006; Lockwood et al., 2006, also submitted manuscript, 2007], that the $I D V$, $I H V$ and $m$ indices have been proposed.

[4] None of these new alternative indices can be used to study the diurnal and seasonal variations at the individual stations. This is disappointing since both the $I D V$ and $I H V$ indices - first to enable computer processing of more than a century of hourly geomagnetic data - indicate that there are significant differences in response by stations at differing geomagnetic latitudes. For these indices a global ring of stations with longitudinal separation may be used to synthesise diurnal responses at either global or latitudinal, but not single station, resolution in a similar manner to that used in Section 6 of this work. For an example, at global resolution, see Svalgaard and Cliver [2007, Figure 4].

[5] However, this will result in coarser latitudinal resolution than if results from individual stations are available and (although not used for the purpose here) becomes impossible in earlier periods when fewer stations were in place. For this reason a new index, modeled on aspects of the $m$ index, is here described: it allows variations in UT and day-of-year values to be analysed at any magnetometer station.

\section{Definition of the New Index}

[6] The $\sigma_{n}^{H}$ index [Finch, 2008] at a magnetometer station is defined as the values of the standard deviation of the hourly average horizontal component, $\mathrm{H}$, of the terrestrial magnetic field over a period $n$ (a whole number multiple of 00 UT to 00 UT days), each single UT hour being treated separately. There will therefore be 24 values for each period of $n$ days at each station.

[7] In this paper we use $\sigma_{28}^{H}$, or its derived averages, exclusively. For each station the H-component data for a single year and UT hour are divided into 1328 -day periods and the standard deviation of the data for that period is computed. The $\sigma_{28}^{H}$ periods begin on 1 January and increment in non-overlapping steps of 28 days. Any excess days are assigned to the final such period.

[8] The advantage of this formulation is that, in addition to the 24 UT bins (which allow studies of the diurnal variations), there are, for $\sigma_{28}^{H}, 13$ time-of-year bins (which allow studies of the seasonal and annual variations). The use of the standard deviations removes effects of zero offset instrument drifts and differences and the need to define baseline quiet days (an advantage for studies of long-term trends).

[9] We further define $\left[\sigma_{n}^{H}\right]$ to be the average over all the UT hours, and $\left\langle\sigma_{n}^{H}\right\rangle_{p}$ to be the separate averages for each UT hour over the period $p$ (where $p$ is a whole multiple of $n$ ). The former will therefore be a single number while the 


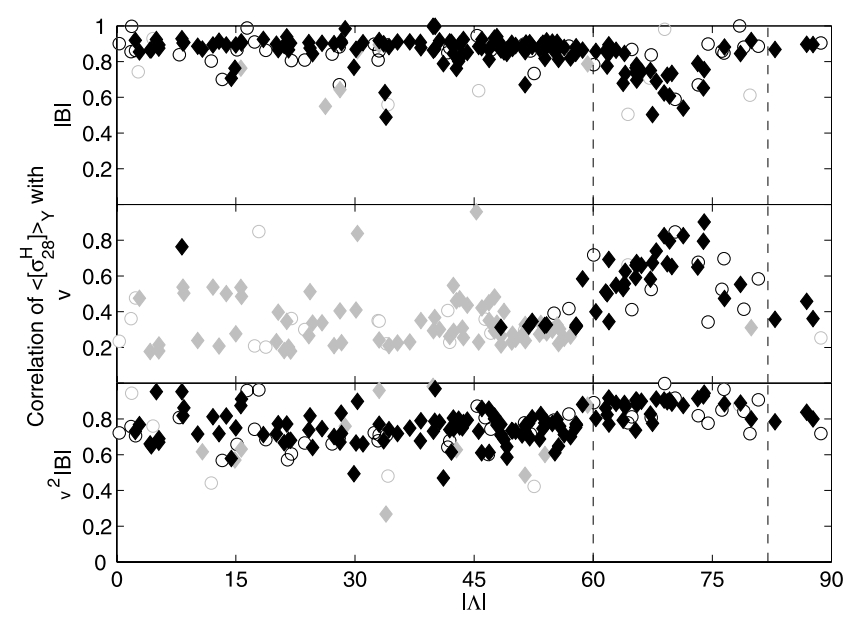

Figure 1. Correlation coefficients of the annual means of the coupling functions (top) $|B|$, (middle) $v$, and (bottom) $v^{2}|B|$ with $\left\langle\left[\sigma_{28}^{H}\right]\right\rangle_{Y}$ shown against absolute geomagnetic latitude. Solid diamonds are for northern hemisphere stations and open circles for southern. Black (grey) for values significant at the $2 \sigma(1 \sigma)$ level. The dashed lines show absolute latitudes of $60^{\circ}$ and $82^{\circ}$.

latter will have 24 values for the extended period $p$. The $m$ index, of Lockwood et al. (submitted manuscript, 2007), in these terms is therefore based on $\left\langle\sigma_{M}^{H}\right\rangle_{Y}$, where $M$ and $Y$ represent a calendar month and year respectively.

\section{Data Sources}

[10] In constructing the $\sigma_{28}^{H}$ indices here, all of the available hourly mean magnetometer data from the World Data Centre (WDC) for Geomagnetism at Edinburgh have been used, along with the geographic and geomagnetic coordinates from the WDC for Geomagnetism at Kyoto. The geomagnetic coordinates are calculated from the International Geomagnetic Reference Field Model (IGRF 10th).

[11] In such a large collection of data (from a total of 270 stations, some containing more than a century of hourly values), some data will inevitably be erroneous. For this reason all hourly values of the $\mathrm{H}$-component which lie more than 6 standard deviations from the average for that station are removed. Analysis shows that this criterion removes most of the data which has been noted by previous authors as being unreliable.

[12] The OMNI 2 data set of hourly-resolution solar wind and IMF data, sourced from a variety of satellites and propagated to the bow-shock nose, is also used. This data set is comprised of a large number of parameters, though in this work only a subset is used, including bulk flow speed, $v$, and interplanetary magnetic field (IMF) strength, $|B|$.

\section{Latitude Dependence of Coupling Correlations}

[13] Figure 1 shows how the zero-lag correlations between $\left\langle\left[\sigma_{28}^{H}\right]\right\rangle_{Y}$ (i.e. $\sigma_{28}^{H}$ averaged over all UT and then averaged over 1 year) and the annual means of the coupling functions $|B|, v$ and $v^{2}|B|$ vary with the absolute geomagnetic latitude, $|\Lambda|$, for all available magnetometer stations. The annual averages of the solar wind parameters are generated from 28 day averages, for exactly the same intervals as described for $\sigma_{28}^{H}$, which are then further averaged over the year. Only correlations significant at the $2 \sigma$ level, shown in black, are discussed unless noted otherwise. A number of dependencies are immediately clear, all of which are mirrored around the geomagnetic equator as demonstrated by the same patterns for the northern and southern stations (solid and open points, respectively).

[14] The correlation with $|B|$ is constant at a level of about 0.86 at all latitudes. The exception is at auroral latitudes where the correlation almost halves at $70^{\circ}$ geomagnetic latitude. This implies that $\sigma_{28}^{H}$ at these latitudes is influenced by another factor which reduces the correlation with $|B|$.

[15] The most distinctive pattern is present in the $v$ plot as a distinct peak centred once more on $70^{\circ}$. At latitudes below about $45^{\circ}$ almost no correlations with $v$ were found that were significant at above the $2 \sigma$ level, instead the $1 \sigma$ results indicate that the correlation is reduced. The origin of this behaviour in both correlations is explored in the next sections. Unfortunately, given the difficulty of reconstructing $v$ before the spacecraft era, none of the stations close to $70^{\circ}$ have data available before 1957. Sodankylä (SOD) and Sitka (SIT), the two stations at auroral latitudes with longrunning data sets, have correlations with $v$ of only $r=0.53$ and 0.39 respectively.

[16] The correlation with $v^{2}|B|$ is also good and less dependent on latitude. There is a slight upward trend with increasing geomagnetic latitude, maximising at $70^{\circ}$, before falling away slightly in the polar cap.

\section{UT Dependence of Coupling Correlations in the Auroral Oval}

[17] Figure 1 shows that the auroral oval has a significant impact on the correlation between $\left\langle\left[\sigma_{28}^{H}\right]\right\rangle_{Y}$ and the single parameter coupling functions. In order to understand the

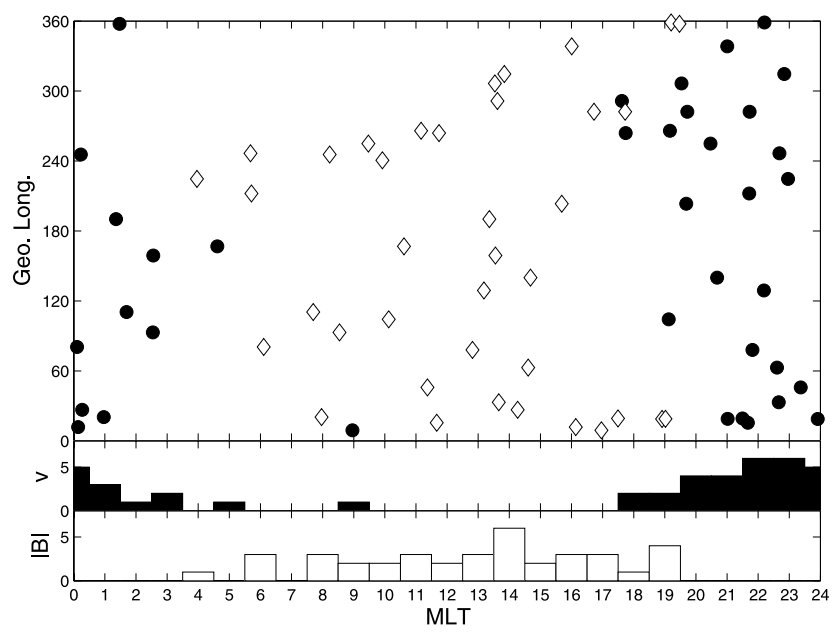

Figure 2. MLT dependence of maximum correlations of $\left\langle\sigma_{28}^{H}\right\rangle_{Y}$ for stations shown in Figure 1 with $60^{\circ}<\Lambda<82^{\circ}$. (top) Filled circles show MLT hour of maximum correlation of $\left\langle\sigma_{28}^{H}\right\rangle_{Y}$ with $v$ and open diamonds show MLT hour of maximum correlation of $\left\langle\sigma_{28}^{H}\right\rangle_{Y}$ with $|B|$. (bottom) Histograms of the MLT hours of the maxima in the correlations for the two coupling functions. 


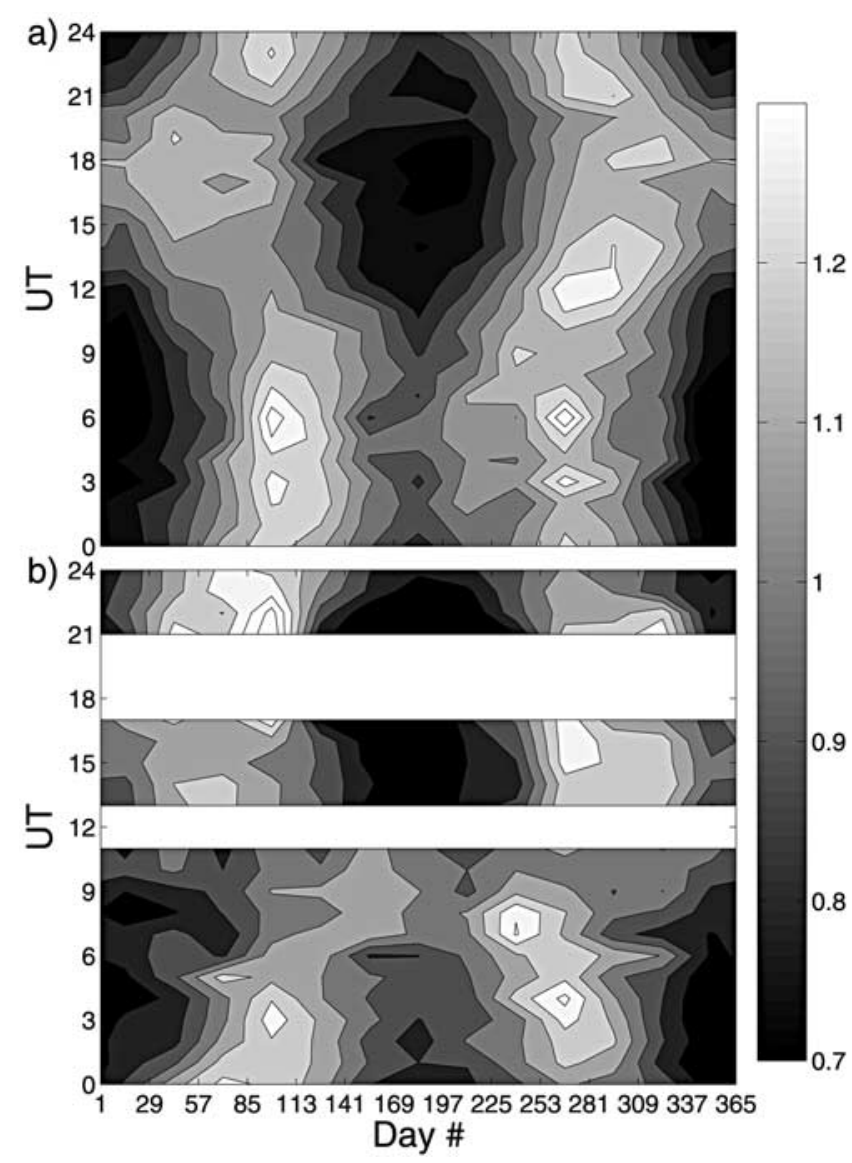

Figure 3. (a) Average of northern hemisphere $\sigma_{28}^{H}$ for postmidnight sectors ( 5 hours centred on 3 MLT) assigned to station UT. (b) Same as Figure 3a for southern hemisphere. Note the data gaps in Figure $3 \mathrm{~b}$ due to insufficient longitude station coverage. The grey scale gives the normalised $\sigma_{28}^{H}$ values, i.e., the average fractional deviation from the overall mean for the contributing stations.

origin of this effect, in Figure 2 (top) the MLT hour of maximum correlation between $v$ and $|B|$ is shown for each of the stations in the auroral oval: the best correlation out of the 24 sequences of $\left\langle\sigma_{28}^{H}\right\rangle_{Y}$ values for each station is used. (A new requirement is imposed that there must be at least five years of coincident spacecraft data.)

[18] Examining the results presented in Figure 2 it is clear that the primary source of the correlation with $v$ occurs in the midnight sector. These are the hours during which the westward electrojet is most likely to be detected. The maximum correlation with $|B|$ can occur at any time except in the midnight sector. Figure 2(bottom) makes this more obvious, showing histograms of the MLT hours of the maxima for correlations with $v$ and $|B|$.

[19] It is thus clear that the correlation with the solar wind velocity at yearly time scales is linked to the storage-release system of the magnetotail and the westward auroral electrojet. In all other locations the correlation is better with $|B|$ (see Figures 1 and 2).

[20] Note that $\sigma_{28}^{H}$, and derived indices, are unable to separate periods of predominantly southward versus northward IMF; for such periods AE [Pulkkinen et al., 2007a] and cusp latitude [Newell et al., 2006] are known to display different short-term couplings with $v$ and $B_{Z}$. Based on MHD simulations Pulkkinen et al. [2007b] argue that even during stationary convection $v$ is a stronger driver than $B_{Z}$, thus relating the processes to magnetotail convection pattern and activity, rather than storage-release cycle per se.

[21] Previous studies have found the best correlation of the westward auroral electrojet, quantified by the $A L$ index, is with $v^{2}|B|$ [e.g., Holzer and Slavin, 1982]. The present study is the first to show that this is the only region where this is the best correlation.

\section{Comparison With AU and AL Observations}

[22] Allen and Kroehl [1975] studied the UT time at which the various $A E$ stations most frequently contributed to the $A L$ and $A U$ indices. They found that " $A L$ was most often derived from records of stations located about 3.25 hours past local geomagnetic midnight", indicating that the westward electrojet effect peaked at this time. Stations were most likely to contribute to $A U$ when "located 6.5 hours before local geomagnetic midnight", i.e. at 17:30 MLT, indicating that the eastward electrojet peaked at this time.

[23] In order to examine the UT variation of the westward and eastward electrojets we separate the hours around 18 MLT and 3 MLT for each station (we here use the 5 hours centred about those times) and assign the data to its corresponding UT hour. The hourly values are self-normalised using the overall mean for that station and then an overall average for each UT hour is computed. The results for the westward electrojet for the northern and southern hemispheres are shown in Figure 3 and for the eastward electrojet in Figure 4. The westward electrojet in both hemispheres show the unmistakeable signal of the equinoctial effect [Bartels, 1925; McIntosh, 1959; Cliver et al., 2000]. The eastward electrojet on the other hand shows no UT variation, instead a simple seasonal variation which maximises in the appropriate hemisphere's summer.

[24] Lyatsky et al. [2001] show similar results for the $A U$ and $A L$ indices but the equinoctial and summer variations are much clearer in these $\sigma_{28}^{H}$ data; this is probably due to the better station coverage when compared to the $A E$ indices. In addition, unlike $A U$ and $A L$, we here show the behaviour is the same in both hemispheres. These results clearly show that the equinoctial effect seen in the highlatitude magnetometer station data originates in the westward auroral electrojet $(A L)$ and hence the substorm current wedge system. The results presented here also confirm a second feature that can be deduced from Lyatsky et al. [2001], namely that the equinoctial effect does not similarly affect the eastward electrojet $(A U)$, which is a signature of the directly driven DP2 current system.

\section{Conclusions}

[25] The $\sigma_{28}^{H}$ indices introduced here for the first time have great advantages for the study of solar-wind/magnetosphere coupling. Reliable data coverage covering 241 hour UT intervals and 13 28-day time-of-year bins are obtained at the level of individual magnetometer stations. This allows latitudinal variations to be studied at the highest resolution. Importantly for the study of long-term variabil- 


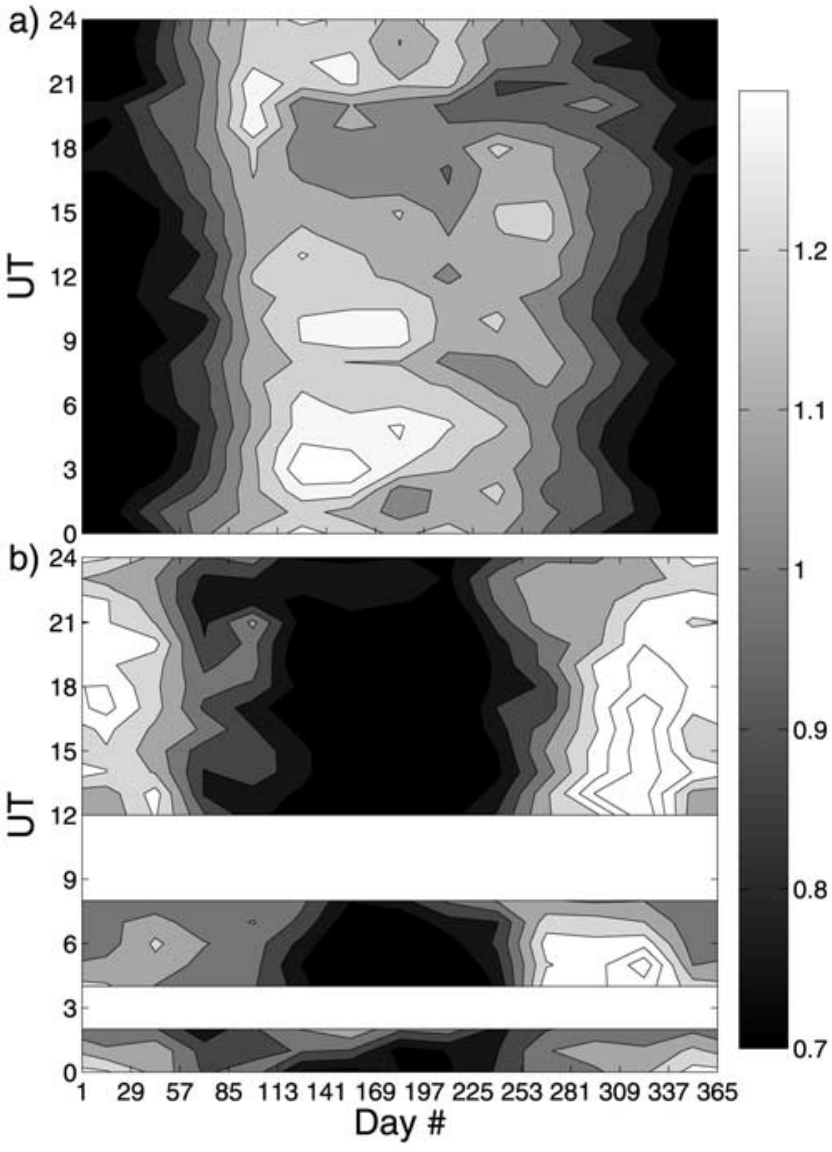

Figure 4. Same as Figure 3 for dusk sectors (5 hours centred on 18 MLT).

ity, the indices can be readily generated from digital data (hourly means).

[26] It is shown that at most latitudes $\left\langle\left[\sigma_{28}^{H}\right]\right\rangle_{Y}$ correlates best with IMF $|B|$, but at auroral latitudes a strong dependence on solar wind speed $v$ occurs. Analysis of the MLT dependency shows that this $v$ dependence is introduced only by data in the midnight sector and so is a feature of the auroral electrojet and the substorm current wedge. At all other MLT and all non-auroral stations the effect of $v$ is not detected and the correlation with $|B|$ dominates. The existing IDV index is similarly independent of $v$. Thus the dependence on $v$ is caused by the storage-release system, giving an optimum correlation with $v^{2}|B|$. Away from the current wedge, better correlations (of the directly driven system) are obtained with $|B|$.

[27] Lastly, the $\sigma_{28}^{H}$ indices confirm the findings from the $A L$ and $A U$ indices that the equinoctial effect is a function of the storage/release system, but that a purely seasonal effect is seen for the directly-driven system. The $\sigma_{28}^{H}$ indices show this result is true for the southern hemisphere as well as the northern (from where $A L$ and $A U$ are derived).

[28] We conclude that the rate of magnetic reconnection at the dayside magnetosphere, responsible for the DP2 (directly-driven) system, does not depend on $v$, nor does it show the equinoctial effect. However, both are found in
DP1 and so are introduced by the storage and release of energy in the tail that is made possible by that reconnection.

[29] Acknowledgments. Magnetometer data were obtained from the World Data Centre for Geomagnetism (Edinburgh) and we also thank Richard Henwood at the UK Solar System Data Centre (UKSSDC) at Rutherford Appleton Laboratory. The OMNI data were obtained from the GSFC/SPDF OMNIWeb interface.

\section{References}

Allen, J. H., and H. W. Kroehl (1975), Spatial and temporal distributions of magnetic effects of auroral electrojets as derived from AE indices, J. Geophys. Res., 80, 3667-3677.

Bartels, J. (1925), Eine universelle Tagsperiode der erdmagnetischen Aktivität, Meteorol. Z., 42, 147.

Cliver, E. W., Y. Kamide, and A. G. Ling (2000), Mountains versus valleys: Semiannual variation of geomagnetic activity, J. Geophys. Res., 105, 2413-2424, doi:10.1029/1999JA900439.

Clilverd, M. A., T. D. G. Clark, E. Clarke, H. Rishbeth, and T. Ulich (2002), The causes of long-term change in the aa index, J. Geophys. Res., 107(A12), 1441, doi:10.1029/2001JA000501.

Clilverd, M. A., E. Clarke, T. Ulich, J. Linthe, and H. Rishbeth (2005), Reconstructing the long-term $a a$ index, J. Geophys. Res., 110, A07205, doi:10.1029/2004JA010762.

Finch, I. D. (2008), The use of geomagnetic activity observations in studies of solar wind-magnetosphere coupling and centennial solar change, Ph.D. thesis, Univ. of Southampton, Southampton, U. K.

Holzer, R. E., and J. A. Slavin (1982), An evaluation of three predictors of geomagnetic activity, J. Geophys. Res., 87, 2558-2562.

Jarvis, M. J. (2005), Observed tidal variation in the lower thermosphere through the 20th century and the possible implication of ozone depletion, J. Geophys. Res., 110, A04303, doi:10.1029/2004JA010921.

Lockwood, M., A. P. Rouillard, I. Finch, and R. Stamper (2006), Comment on "The IDV index: Its derivation and use in inferring long-term variations of the interplanetary magnetic field strength" by Leif Svalgaard and Edward Cliver, W., J. Geophys. Res., 111, A09109, doi:10.1029/ 2006JA011640.

Lyatsky, W., P. T. Newell, and A. Hamza (2001), Solar illumination as cause of the equinoctial preference for geomagnetic activity, Geophys. Res. Lett., 28, 2353-2356.

Martini, D., and K. Mursula (2006), Correcting the geomagnetic IHV index of the Eskdalemuir observatory, Ann. Geophys., 24, 3411-3419.

Mayaud, P. N. (1980), Derivation, Meaning, and Use of Geomagnetic Indices, Geophys. Monogr. Ser, vol. 22, AGU, Washington, D. C.

McIntosh, D. H. (1959), On the annual variation of magnetic disturbance, Philos. Trans. R. Soc. London, Ser. A, 251, 525-552.

Newell, P. T., T. Sotirelis, K. Liou, C.-I. Meng, and F. J. Rich (2006), Cusp latitude and the optimal solar wind coupling function, J. Geophys. Res., 111, A09207, doi:10.1029/2006JA011731.

Pulkkinen, T. I., M. Palmroth, and R. L. McPherron (2007a), What drives magnetospheric activity under northward IMF conditions?, Geophys. Res. Lett., 34, L18104, doi:10.1029/2007GL030619.

Pulkkinen, T. I., C. C. Goodrich, and J. G. Lyon (2007b), Solar wind electric field driving of magnetospheric activity: Is it velocity or magnetic field?, Geophys. Res. Lett., 34, L21101, doi:10.1029/2007GL031011.

Rouillard, A. P., M. Lockwood, and I. Finch (2007), Centennial changes in the solar wind speed and in the open solar flux, J. Geophys. Res., 112, A05103, doi:10.1029/2006JA012130.

Svalgaard, L., and E. W. Cliver (2005), The IDV index: Its derivation and use in inferring long-term variations of the interplanetary magnetic field strength, J. Geophys. Res., 110, A12103, doi:10.1029/2005JA011203.

Svalgaard, L., and E. W. Cliver (2007), Interhourly variability index of geomagnetic activity and its use in deriving the long-term variation of solar wind speed, J. Geophys. Res., 112, A10111, doi:10.1029/ 2007JA012437.

Svalgaard, L., E. W. Cliver, and P. LeSager (2004), IHV: A new long-term geomagnetic index, Adv. Space Res., 34(2), 436-439, doi:10.1016/ j.asr.2003.01.029.

I. D. Finch and M. L. Lockwood, Space Science and Technology Department, Rutherford Appleton Laboratory, Oxfordshire OX11 0QX, UK. (i.d.finch@rl.ac.uk; m.lockwood@rl.ac.uk)

A. P. Rouillard, Solar-Terrestrial Physics Group, Department of Physics and Astronomy, University of Southampton, Southampton SO17 1BJ, UK. (alexisrouillard@yahoo.co.uk) 